\title{
Um Teorema de Periodicidade para uma Classe de Equações de Segunda Ordem com Retardamento
}

\author{
Rita de Cássia Pavani Lamas
}

\author{
Orientação \\ Prof. Dr. Plácido Zoéga Táboas
}

Dissertação apresentada ao Instituto de Ciências Matemáticas de São Carlos USP, como parte dos requisitos para obtenção do título de Mestre em Matemática.

USP - São Carlos

julho de 1991 
Ao meu esposo,

Décio Lamas.

Aos meus filhos,

Tiago e André. 
Quero agradecer a todos que direta ou indiretamente contribuíram para que esse trabalho fosse realizado. Especialmente agradeço ao professor Plácido por sua orientação segura e precisa, por sua dedicação, incentivo e amizade; ao meu esposo, pela compreensão, dedicação e apoio recebidos e pelos esforços feitos para que eu conseguisse concluir mais uma etapa de minha vida; aos meus filhos, pela compreensão; aos docentes e técnicos do ICMSC-USP, pelo apoio recebido durante esses anos de mestrado; aos amigos Kasuo e Maria do Carmo, pela amizade e ajuda nos problemas de edição deste trabalho; a todos os amigos da pós-graduação que sempre me deram apoio, amizade; e ao $\mathrm{CNPq}$ pelo auxílio financeiro recebido. 


\begin{abstract}
We are concerned with the retarded second order differential equation

$$
\ddot{x}(t)+f(x(t)) \dot{x}(t)+g(x(t-r))=0 .
$$

Our aim consists in following the flow given by (E) to define a return map $A$ on a closed convex set of the phase space, in order to study the periodic solutions.

For a small delay $r$, we use a fixed point theorem due to R. Nussbaum, Theorem(1.5) below, to accomplish the existence of nonconstant periodic solutions of (E), which correspond to nontrivial fixed points of $A$.
\end{abstract}




\section{Resumo}

Estamos interessados na equação diferencial retardada de segunda ordem

$$
\bar{x}(t)+f(x(t)) \dot{x}(t)+g(x(t-r))=0 .
$$

Nosso objetivo consiste em, seguindo o fluxo dado por (E), definir uma aplicação de retorno $A$ sobre um conjunto fechado convexo do espaço de fase, para estudar as soluções periódicas.

Para um retardamento $r$ suficientemente pequeno, usamos um teorema de R. Nussbaum, veja Teorema(1.5), para obter a existência de soluções periódicas não constante de (E), as quais correspondem a pontos fixos não triviais de $A$. 


\section{Conteúdo}

1 Definições e Fatos Básicos $\quad 1$

$\begin{array}{lll}2 & \text { Linearização e Bifurcação de Hopf } & 7\end{array}$

3 Um Teorema de Periodicidade $\quad 16$ 


\section{Introdução}

O objetivo deste trabalho é mostrar a existência de soluções periódicas da equação diferencial retardada de segunda ordem

$$
\ddot{x}(t)+f(x(t)) \dot{x}(t)+g(x(t-r))=0
$$

onde $x$ é real, $f(0)=-k$ e $f, g$ estão sob certas condições.

No primeiro Capítulo, apresentamos algumas definições e fatos básicos que achamos oportuno relembrar.

No segundo Capítulo, mostramos que a equação acima tem uma Bifurcação de Hopf para um valor do parâmetro $k$. Ainda nesse Capítulo para um retardamento $r$ pequeno, alguns resultados que dependem do parâmetro $k$, foram extendidos para valores, não positivos de $k$, sendo que em Hale [4] esses resultados são obtidos apenas para $k$ positivo.

Finalizando, mostramos que a equação (E) possui de fato uma solução periódica não constante, para $k$ percorrendo um intervalo $[a, b]$, onde $a<0$ e $b>0$.

A equação (E) pode ser entendida como a equação de um oscilador, onde o termo $f(x(t)) \dot{x}(t)$ pode representar uma dissipação de energia (atrito) e a função $g$ a força restauradora. $O$ retardamento $r$ indica que essa força não responde imediatamente aos estímulos. Há um lapso de tempo $r$ entre a ação e a reação. Essa característica da força restauradora pode parecer estranha, pensando em modelos oriundos da Física Clássica. Entretanto, em se tratando de aplicações à Biologia, Ecologia, Epidemiologia, etc., essa é uma hipótese bastante realística. 
Em sistemas dessa natureza há sempre um tempo de reação, um período de incubação, etc. Por esta razão as Equações com Retardamento encontram grande sucesso em aplicações à essas ciências. 


\section{Capítulo 1}

\section{Definições e Fatos Básicos}

Consideremos $r \geq 0$ um número real dado, o espaço vetorial $n$-dimensional $\mathbf{R}^{n}$ sobre os reais com a norma euclidiana $||,. C\left([a, b], \mathbf{R}^{n}\right)$ o espaço de Banach das aplicações contínuas do intervalo $[a, b]$ tomando valores em $\mathbf{R}^{n}$, munido da norma do supremo.

Seja $[a, b]=[-r, 0]$ e $C=C\left([-r, 0], \mathbf{R}^{n}\right)$, onde a norma de um elemento $\phi$ $\in C$ é dada por

$$
|\phi|=\sup _{-r \leq \theta \leq 0}|\phi(\theta)| .
$$

Definição 1.1 Sejam $\sigma \in \mathbf{R}, A \geq 0$ e $x \in C\left([\sigma-r, \sigma+A], \mathbf{R}^{n}\right)$. Para cada $t \in[\sigma, \sigma+A]$, definimos a função $x_{t} \in C$ por

$$
x_{t}(\theta)=x(t+\theta),
$$

onde $-r \leq \theta \leq 0$.

Definição 1.2 Sejam $D$ um subconjunto de $\mathbf{R} \times C, f: D \rightarrow \mathbf{R}^{n}$ uma função, com ". " representando a derivada à direita. A equação

$$
\dot{x}=f\left(t, x_{t}\right)
$$

é dita uma equação diferencial funcional retardada sobre $D$ e será denotada por $\operatorname{EDFR}(f)$. 
Definição 1.3 Se existe $\sigma \in \mathbf{R}$, e $A>0$ tal que $x \in C\left([\sigma-r, \sigma+A), \mathbf{R}^{n}\right)$, $\left(t, x_{t}\right) \in D$ e $x(t)$ satisfaz à equação (1.1) para $t \in[\sigma, \sigma+A)$, diz se que $x$ é solução da equação (1.1).

Definição 1.4 Dado $\sigma \in \mathbf{R}, \phi \in C$, dizemos que $x(\sigma, \phi, f)$ é solução da equação (1.1) com valor inicial $\phi$ em $\sigma$ se :

1. existe $A>0$ tal que $x(\sigma, \phi, f)$ é solução da equação (1.1) sobre $[\sigma-r, \sigma+A)$, 2. $\mathrm{x}_{\boldsymbol{\sigma}}(\sigma, \phi, f)=\phi$.

A teoria das equações diferenciais funcionais retardadas é inspirada na teoria das equações diferenciais ordinárias, as quais são um tipo particular da equação (1.1), bastando tomar $r=0$.

É importante ressaltar que o Teorema de Existência e Unicidade, do problema de valor inicial e propriedades de extensão de soluções são válidos, como para as equações diferenciais ordinárias, com a ressalva de serem estabelecidos à direita.

Os conceitos acima foram extraídos de Hale [4]. No que segue estaremos também admitindo alguns fatos fundamentais da teoria geral contida em Hale [4].

Definição 1.5 Sejam $X$ um espaço de Banach, $U$ um subconjunto de $X$, e $x$ um ponto de U. Dada uma aplicação $A: U \backslash\{x\} \rightarrow X$, o ponto $x \in U$ é dito um ponto ejetivo de $A$ se há uma vizinhança aberta $G \subseteq X$ de $x$, tal que para cada $y \in$ $G \cap U, y \neq x$, há um inteiro $m=m(y)$ tal que $A^{m} y \notin G \cap U$.

Exemplos: Seja $X=\mathbf{R}$ e $U=[0,1]$. Se $A: U \backslash\{0\} \rightarrow X$ com $A x=x^{1 / 2}$, então 0 é ponto ejetivo de $A$.

Se $A x=x^{2}, 0$ não é ponto ejetivo de $A$. 
Definição 1.6 Sejam $X, Y$ espaços de Banach e $f: A \subset X \rightarrow Y$. A função $f$ é completamente contínua se é contínua e leva cada limitado de $A$ em um conjunto relativamente compacto de $Y$.

Seja $K$ um espaço métrico compacto e $M$ um espaço métrico . Consideremos $C(K, M)$ o conjunto das aplicações contínuas de $\mathrm{K}$ em $\mathrm{M}$ com a topologia dada por

$$
\mathrm{d}(f, g)=\max _{x \in K} \mathrm{~d}(f(x), g(x))
$$

onde $f, g \in C(K, M)$.

Definição 1.7 Um conjunto $E \subset C(K, M)$ é equicontínuo se para todo $\epsilon>0$ existe $\delta>0, \delta=\delta(\epsilon)$ tal que se $d(x, y)<\delta$, então $d(f(x), f(y))<\epsilon, \forall f \in E$.

Teorema 1.1 Todas as raizes da equação $\left(z^{2}+a z\right) e^{z}+1=0$ têm parte real negativa se,e somente se, $a>(\operatorname{sen} \xi) / \xi$ onde $\xi$ é a única raiz da equação $\xi^{2}=$ $\cos \xi, 0<\xi<\pi / 2$.

Daremos a seguir uma versão do teorema de Rouché.

Teorema 1.2 (Rouché) Se $f(z)$ e $g(z)$ são analíticas sobre o fecho da região limitada por uma curva simples $C e|g(z)|<|f(z)|$ para todo $z \in C$, então $f(z)+$ $g(z)$ e $f(z)$ têm os mesmos números de zeros nessa região. [Conway [2]]

Consideremos uma família a um parâmetro de EDFRs da forma

$$
\dot{x}(t)=F\left(\alpha, x_{t}\right),
$$

onde $F(\alpha, \phi)$ tem primeira e segunda derivadas contínuas em $\alpha, \phi$, para $\alpha \in \mathbf{R}$, $\phi \in C$, e $F(\alpha, 0)=0$ para todo $\alpha$. 
Definição 1.8 Seja $L: \mathbf{R} \times C \rightarrow \mathbf{R}^{n}$ dada por

$$
L(\alpha) \psi=F_{\phi}(\alpha, 0) \psi,
$$

onde $F_{\phi}(\alpha, 0)$ é a derivada de $F(\alpha, \phi)$ com respeito a $\phi$ em $\phi=0$.

Considere as seguintes hipóteses:

(H1) A $\operatorname{EDFR}(L(0))$ linear tem uma raiz característica imaginária pura simples $\lambda_{0}=i v_{0} \neq 0$ e toda raiz característica $\lambda_{j} \neq \lambda_{0}, \overline{\lambda_{0}}$, satisfaz $\lambda_{j} \neq m \lambda_{0}$ para todo inteiro $m$.

De acordo com [4, Lemma 2.2, sect. 7.2], os autovalores $\lambda(\alpha)$ da $\operatorname{EDFR}(L(\alpha))$ próximos de $\lambda_{0}$, são simples e dependem diferenciavelmente de $\alpha$ numa vizinhança de $\alpha=0$.

(H2) $\Re\left(\lambda^{\prime}\right)(0) \neq 0$, onde a linha representa a derivada de $\lambda$ em relação a $\alpha$.

O Teorema enunciado a seguir é conhecido como Teorema de Bifurcação de Hopf .

Teorema 1.3 Suponhamos $F(\alpha, \phi)$ continuamente diferenciável com respeito a $\alpha, \phi, F(\alpha, 0)=0$ para todo $\alpha, e$ as hipóteses (H1) e (H2) satisfeitas. Então existem constantes $a_{0}>0, \alpha_{0}>0, \delta_{0}>0$, funções continuamente diferenciáveis $\alpha(a) \in \mathbf{R}, \omega(a) \in \mathbf{R}$ e $x^{*}(a) \omega(a)$ - periódica, para $|a|<a_{0}$, tal que $x^{*}(a)$ é uma soluçâo da equação (1.2). Além disso, para $|\alpha|<\alpha_{0},\left|\omega-\left(2 \pi / v_{0}\right)\right|<\delta_{0}$, as soluções $x^{*}$ são as únicas $\omega$ - periódicas da equação (1.2) com $\left|x_{t}\right|<\delta_{0}$, exceto por uma translação de fase.

Daremos a seguir uma versão do teorema de Ascoli útil aos nossos propósitos.

Teorema 1.4 (Ascoli-Arzela) Sejam $K$ um espaço métrico compacto $e$ $E \subset C\left(K, \mathbf{R}^{m}\right) . S e$

1. existe $N \geq 0$ tal que $|f(x)| \leq N, \forall x \in K, \forall f \in E$, 


\section{E é equicontínuo,}

então E é relativamente compacto.

Definição 1.9 Dado $M>0$, definimos $S_{M}=\{x \in X:|x|=M\}$ e $B_{M}=$ $\{x \in X:|x|<M\}$.

Observemos que $S_{M}=\partial B_{M}$.

O Teorema abaixo é atribuido a R.Nussbaum [5]

Teorema 1.5 Sejam $X$ um espaço de Banach, $K$ um subconjunto convexo $e$ fechado de $X$ e $A: K \backslash\{0\} \rightarrow K$ uma aplicação completamente contínua, tal que $0 \in K$ é um ponto ejetivo de A. Suponhamos que exista um $M>0$ tal que $A x=\lambda x$ implique $\lambda<1$ para todo $x \in K \cap S_{M}$. Então $A$ tem um ponto fixo em $K \cap B_{M} \backslash\{0\}$ se $K$ tem dimensão infinita ou 0 é um ponto extremo de $K$.

Consideremos as equações diferenciais retardadas

$$
\begin{gathered}
\dot{x}(t)=L x_{t}+f\left(x_{t}\right) \\
\dot{y}(t)=L y_{t},
\end{gathered}
$$

onde $\mathrm{L}: \mathrm{C} \rightarrow \mathbf{R}^{2}$ é uma aplicacão linear contínua, $f$ é completamente contínua com sua primeira derivada contínua , $f(0)=0$ e $D f(0)=0$. A equação característica da equação (1.4) é dada por

$$
\operatorname{det} \Delta(\lambda)=0,
$$

onde $\Delta(\lambda)=\lambda I-L\left(e^{\lambda} I\right)$. Suas raízes serão chamadas indistintamente raizes características ou autovalores.

Para cada autovalor $\lambda$, o espaço de fase $C$ é decomposto como soma direta, $C=P_{\lambda} \oplus Q_{\lambda}$, onde $P_{\lambda}$ e $Q_{\lambda}$ são invariantes sob o operador solução da equação (1.4), $T(\mathrm{t}), t \geq 0$ dado por $T(t) \phi=y_{t}(., \phi), \phi \in C \mathrm{e} \pi_{\lambda}$ o operador projeção com imagem $P_{\lambda}$ definido por essa decomposição. 
Teorema 1.6 Suponhamos que as seguintes condições estejam satisfeitas:

1. Há um autovalor $\lambda$ da equação (1.4) com $\Re(\lambda)>0$.

2. Há um subconjunto convexo fechado $K$ de $C, 0 \in K$, e uma função contínua $\tau: K \backslash\{0\} \rightarrow[\rho, \infty), \rho>0$, tal que a aplicação $A: K \rightarrow C$ dada por

$$
\begin{aligned}
A \phi & =x_{\tau(\phi)}(\cdot ; \phi), \phi \in K \backslash\{0\} \\
A 0 & =0,
\end{aligned}
$$

é completamente contínua e $A K \subset K$.

3. $\inf \left\{\left|\pi_{\lambda} x_{t}\right|: x_{t}=x_{t}(. ; \phi), \phi \in K,|\phi|=\delta, \delta>0,0 \leq t \leq \tau(\phi)\right\}>0$.

4. Dado $G \subset C$ aberto, $0 \in G$, há uma vizinhança $V$ de 0 tal que $x_{t}(. ; \phi) \in G$, se $\phi \in V \cap K, \phi \neq 0, e 0 \leq t \leq \tau(\phi)$.

Então 0 é um ponto ejetivo de A. 


\section{Capítulo 2}

\section{Linearização e Bifurcação de Hopf}

Nosso objetivo nesse capítulo, é mostrar a existência de soluções periódicas não constantes da equação

$$
\ddot{x}(t)+f(x(t)) \dot{x}(t)+g(x(t-r))=0
$$

onde $x$ é real, $r \geq 0, f$ e $g$ são continuamente diferenciáveis, $f(0)=-k, g^{\prime}(0)=1$ com $x g(x)>0$.

Em primeiro lugar considerando $k$ como um parâmetro, mostraremos que a equação (2.1) tem uma Bifurcação de Hopf em $k=-k_{0}$, onde $k_{0}$ é um valor a ser definido precisamente no que se segue. Em seguida, utilizando os teoremas enunciados no Capítulo 1, mostraremos que de fato existe solução periódica não constante da equação (2.1) para $k>-k_{0}$.

Vamos obter a equação característica da parte linear da equação (2.1). Por Taylor, em uma vizinhança da origem temos

$$
f(x)=-k+f^{\prime}(0) x+o\left(x^{2}\right) \text { e } g(x)=x+o\left(x^{2}\right)
$$

Substituindo na equação (2.1) temos;

$$
\ddot{x}(t)+\left(-k+f^{\prime}(0) x(t)+o\left(x^{2}\right)\right) \dot{x}(t)+\left(x(t-r)+o\left(x^{2}\right)\right)=0 .
$$


Logo a parte linear é

$$
\ddot{x}(t)-k \dot{x}(t)+x(t-r)=0 .
$$

Assim, uma condição necessária e suficiente para a existência de uma solução não trivial da equação (2.2) da forma $x(t)=e^{\lambda t} u, u \in \mathbf{R} \backslash\{0\}$ é que $u$ seja uma solução não nula de

$$
\lambda^{2} e^{\lambda t} u-k \lambda e^{\lambda t} u+e^{\lambda(t-r)} u=0
$$

ou seja,

$$
\left(\lambda^{2}-k \lambda+e^{-\lambda r}\right) u=0,
$$

a qual nos leva à equação característica da equação (2.2),

$$
\lambda^{2}-k \lambda+e^{-\lambda r}=0
$$

Lema 2.1 Seja $S=\left\{\lambda \mid \lambda^{2}-k \lambda+e^{-\lambda r}=0 ; k \in\left[k_{1}, k_{2}\right]\right\}$. Se a sequência $\lambda_{j} \in S, j=1,2, \ldots$, é tal que $\left|\lambda_{j}\right| \rightarrow \infty$ quando $j \rightarrow \infty$, então

1. $\Re\left(\lambda_{j}\right) \rightarrow-\infty$ quando $j \rightarrow \infty$,

2. existe $b>0$ tal que $S_{b}=\{\lambda \in S: \Re(\lambda) \geq b\}$ é limitado e finito.

Prova: 1 . Se $\lambda_{j} \in S$, então

$$
\lambda_{j}^{2}-k \lambda_{j}+e^{-\lambda_{j} r}=0
$$

donde

$$
\lambda_{j}^{2}-k \lambda_{j}=-e^{-\lambda_{j} r}
$$

ou seja,

$$
\left|\lambda_{j}\right|\left|\lambda_{j}-k\right|=e^{-\Re\left(\lambda_{j}\right) r}
$$

onde $k \in\left[k_{1}, k_{2}\right]$. Temos que $\left|\lambda_{j}\right|\left|\lambda_{j}-k\right| \rightarrow \infty$, quando $j \rightarrow \infty$. 
Logo, da última equação

$$
\Re\left(\lambda_{j}\right) \rightarrow-\infty
$$

2. Suponhamos que para todo $b>0, S_{b}$ é ilimitado. Então existe sequência $\lambda_{j} \in S$ com $\left|\lambda_{j}\right| \rightarrow \infty$ tal que $\Re\left(\lambda_{j}\right) \rightarrow \infty$, contrariando (1).

Se $S_{b}$ fosse infinito teria um ponto de acumulação o que é uma contradição com o fato de $\lambda^{2}-k \lambda+e^{-\lambda r}$ ser uma função analítica não constante.

Concluimos com isto que existe um número finito de raízes, $\operatorname{com} \Re(\lambda) \geq b$.

Lema 2.2 Seja $\sigma_{=} \sigma_{0}(r), 0<\sigma_{0}<\pi / 2 r$, a única solução de $\sigma^{2}=\cos \sigma r$, e $k_{0}(r)=\left[\sigma_{0}\right]^{-1} \operatorname{sen} \sigma_{0} r$.

1. Se $k<-k_{0}(r)$ então todas as raizes da equação (2.3) tem parte real negativa.

2. Existe um $\epsilon>0$ e uma raiz $\lambda(k)$ da equação (2.3) a qual é continuamente diferenciável em $k$ para $k \in\left(-k_{0}(r)-\epsilon,-k_{0}(r)+\epsilon\right)$; $\operatorname{com} \lambda\left(-k_{0}(r)\right)=i \sigma_{0}$.

3. $\Re\left(\lambda^{\prime}\left(-k_{0}(r)\right)\right)>0$.

4. Para cada $k>-k_{0}(r)$, existem precisamente duas raízes $\lambda$ da equação (2.3) $\operatorname{com} \Re(\lambda)>0$ e $-\pi / r<\Im(\lambda)<\pi / r$.

Prova: Suponhamos que $\lambda=\mu+i \sigma$, onde $\mu=\mu(r)$ e $\sigma=\sigma(r)$, é uma solução da equação (2.3). Assim,

$(\mu+i \sigma)^{2}-k(\mu+i \sigma)+e^{-(\mu+i \sigma) r}=\mu^{2}+2 i \mu \sigma-\sigma^{2}-k \mu-i k \sigma+e^{-\mu r}(\cos \sigma r-i \operatorname{sen} \sigma r)=0$, ou seja,

$$
\left\{\begin{array}{l}
\mu^{2}-\sigma^{2}-k \mu+e^{-\mu r} \cos (\sigma r)=0 \\
2 \mu \sigma-k \sigma-e^{-\mu r} \operatorname{sen}(\sigma r)=0
\end{array}\right.
$$

1. É uma adaptação do Teorema (1.1). 
2. Se $k=-k_{0}(r)$, temos que $\mu=0$ e $\sigma=\sigma_{0}$ são soluções da equação (2.4) e, como a matriz jacobiana

$$
\left(\begin{array}{ll}
\left(2 \mu-k-r e^{-\mu r} \cos \sigma r\right) & \left(-2 \sigma-r e^{-\mu r} \operatorname{sen} \sigma r\right) \\
\left(2 \sigma+r e^{-\mu r} \operatorname{sen} \sigma r\right) & \left(2 \mu-k-r e^{-\mu r} \cos \sigma r\right)
\end{array}\right)
$$

é não singular, podemos aplicar o Teorema da Função Implícita à essa equação. Assim, temos que existe $\epsilon>0$ e uma única solução $\mu(k), \sigma(k) ; \mu\left(-k_{0}(r)\right)=0$, $\sigma\left(-k_{0}(r)\right)=\sigma_{0}$, para $k \in\left(-k_{0}(r)-\epsilon,-k_{0}(r)+\epsilon\right)$, a qual é continuamente diferenciável em $k$.

3. Diferenciando a equação (2.4) com relação a $k$, temos

$$
\left\{\begin{array}{l}
2 \mu \mu^{\prime}-2 \sigma \sigma^{\prime}-\mu-k \mu^{\prime}-r \mu^{\prime} e^{-\mu r} \cos \sigma r-r \sigma^{\prime} e^{-\mu r} \operatorname{sen} \sigma r=0 \\
2 \sigma \mu^{\prime}+2 \sigma^{\prime} \mu-\sigma-k \sigma^{\prime}+r \mu^{\prime} e^{-\mu r} \operatorname{sen} \sigma r-r \sigma^{\prime} e^{-\mu r} \cos \sigma r=0 .
\end{array}\right.
$$

Para $k=-k_{0}(r)$, temos $\mu=0$ e $\sigma=\sigma_{0}$. Assim temos;

$$
\left\{\begin{array}{l}
-2 \sigma_{0} \sigma^{\prime}+k_{0} \mu^{\prime}-\mu^{\prime} \cos \sigma_{0} r-r \sigma^{\prime} \operatorname{sen} \sigma_{0} r=0 \\
2 \sigma_{0} \mu^{\prime}-\sigma_{0}+k_{0} \sigma^{\prime}+r \mu^{\prime} \operatorname{sen} \sigma_{0} r-r \sigma^{\prime} \cos \sigma r=0,
\end{array}\right.
$$

ou ainda,

$$
\left(\begin{array}{cc}
\left(k_{0}-r \cos \sigma_{0} r\right) & \left(-2 \sigma_{0}-r \operatorname{sen} \sigma_{0}\right) \\
\left(2 \sigma_{0}+r \operatorname{sen} \sigma_{0} r\right) & \left(k_{0}-r \cos \sigma_{0} r\right)
\end{array}\right)\left(\begin{array}{c}
\mu^{\prime} \\
\sigma^{\prime}
\end{array}\right)=\left(\begin{array}{c}
0 \\
\sigma_{0}
\end{array}\right) .
$$

O determinante da matriz $2 \times 2$ acima é

$$
\Lambda=\left(k_{0}-r \cos \sigma_{0} r\right)^{2}+\left(2 \sigma_{0}+r \operatorname{sen} \sigma_{0} r\right)^{2} .
$$

Uma vez que $0<\sigma_{0}<\pi / 2 r$, segue - se que $\left(2 \sigma_{0}+r \operatorname{sen} \sigma_{0} r\right)$ é positivo, ou seja, $\Lambda$ é positivo. Logo, sua matriz é inversível.

Dessa maneira, temos

$$
\begin{gathered}
\left(\begin{array}{c}
\mu^{\prime} \\
\sigma^{\prime}
\end{array}\right)=\frac{1}{\Lambda}\left(\begin{array}{ll}
k_{0}-r \cos \sigma_{0} r & 2 \sigma_{0}+r \operatorname{sen} \sigma_{0} r \\
-2 \sigma_{0}-r \operatorname{sen} \sigma_{0} r & k_{0}-r \cos \sigma_{0} r
\end{array}\right)\left(\begin{array}{c}
0 \\
\sigma_{0}
\end{array}\right) \\
\left(\begin{array}{c}
\mu^{\prime} \\
\sigma^{\prime}
\end{array}\right)=\frac{1}{\Lambda}\left(\begin{array}{c}
\left(2 \sigma_{0}+r \operatorname{sen} \sigma_{0} r\right) \sigma_{0} \\
\left(k_{0}-r \cos \sigma_{0} r\right) \sigma_{0}
\end{array}\right) .
\end{gathered}
$$


Portanto,

$$
\mu^{\prime}\left(-k_{0}\right)=\left(2 \sigma_{0}+r \operatorname{sen} \sigma_{0} r\right) \sigma_{0}>0 .
$$

4. Seja $U_{a b}=\{\lambda \in C: a \leq \Re(\lambda) \leq b ;|\Im(\lambda)|<\pi / r\}$. Olhemos para $U_{0 \infty}$.

Para $k<-k_{0}$, todas as raízes de (2.3) possuem parte real negativa; portanto não pertecem á $U_{0 \infty}$. Como na prova do ítem 2, pelo Teorema da Função Implícita garantimos que existe $\epsilon>0$ e uma única solução $\mu(k), \sigma(k)$ da equação (2.4) para $k \in\left(-k_{0}-\epsilon,-k_{0}+\epsilon\right)$, e como $\lambda$ ser raiz da equação (2.3) implica que seu conjugado também é, temos que existe $k_{1}>-k_{0}$, tal que, para cada $k \in\left[-k_{0}, k_{1}\right]$, há exatamente 2 raízes em $U_{0 \infty}$.

Suponhamos agora $k \geq k_{1}$. Mostremos que $\lambda=\mu+i \pi / r, \mu \geq 0$ ou $\lambda=$ $i \sigma, 0 \leq \sigma \leq \pi / r$, não são soluções da equação (2.3). Suponhamos que $\lambda=i \sigma$; $0 \leq \sigma \leq \pi / r$ fosse solução da equação (2.3). Assim, por (2.4) teríamos

$$
\left\{\begin{array}{l}
-\sigma^{2}+\cos \sigma r=0 \\
-k \sigma-\operatorname{sen} \sigma r=0
\end{array}\right.
$$

ou seja,

$$
\left\{\begin{array}{l}
\sigma^{2}=\cos \sigma r \\
k=-(\operatorname{sen} \sigma r) / \sigma .
\end{array}\right.
$$

Para $0<\sigma \leq \pi / r$, temos que $\sigma=\sigma_{0}$ e $k=-k_{0}$ o que é uma contradição com o fato que $k \geq k_{1}>-k_{0}$. Para $\sigma \in(\pi / 2 r, \pi / r], \cos \sigma r<0$, enquanto $\sigma^{2}>0$.

Suponhamos agora que $\lambda=\mu+i \pi / r, \mu \geq 0$ fosse solução da equação (2.3). Pela equação (2.4) temos

$$
\left\{\begin{array}{l}
\mu^{2}-(\pi / r)^{2}-k \mu+e^{-\mu r} \cos \pi=0 \\
\mu=k / 2
\end{array}\right.
$$

Substituindo $\mu$ na primeira equação do sistema (2.5), temos

$$
0=\frac{k^{2}}{4}-\frac{\pi^{2}}{r^{2}}-\frac{k^{2}}{2}-e^{-\frac{k}{2} r}=\frac{-k^{2}}{4}-\left(\frac{\pi}{r}\right)^{2}-e^{-\frac{k}{2} r}<0,
$$

o que dá uma contradição. 
Portanto, $\lambda=\mu+i \pi / r, \mu \geq 0$ e $\lambda=i \sigma, 0 \leq \sigma \leq \pi / r$ não são raízes da equação (2.4) para $k \geq k_{1}$.

Pelo Lema (2.1), ítem 2, para cada $k_{2} \in \mathbf{R}$ fixo, $k_{2}>k_{1}$, há um número real $b\left(k_{2}\right)>b$, tal que não há solução $\lambda$ da equação $(2.3)$ com $\Re(\lambda) \geq b\left(k_{2}\right)$, para $k \in\left[k_{1}, k_{2}\right]$.

Portanto, acabamos de mostrar que, para $k \in\left[k_{1}, k_{2}\right]$, não há solução da equação (2.3) sobre a fronteira de $U_{0 b\left(k_{2}\right)}$.

Seja

$$
f_{t}(\lambda)=\lambda^{2}-\left[(1-t) k_{1}+t k\right] \lambda+e^{-r \lambda}, 0 \leq t \leq 1, k \in\left[k_{1}, k_{2}\right] .
$$

Como $(1-t) k_{1}+t k \in\left[k_{1}, k_{2}\right]$, com $k \in\left[k_{1}, k_{2}\right]$ e $t \in[0,1]$, acabamos de mostrar que, para todo $t \in[0,1], f_{t}$ não tem zeros sobre a fronteira de $U_{0 b\left(k_{2}\right)}$. Assim sendo, tem sentido comparar o número de zeros de $f_{0}$ com o número de zeros de $f_{1}$ na região $U_{0 b\left(k_{2}\right)} \operatorname{com} k \in\left[k_{1}, k_{2}\right]$.

Mostremos usando o Teorema (1.2) que esse número é constante para qualquer $t \in[0,1]$.

Tomemos

$$
\begin{aligned}
& f_{t_{1}}(\lambda)=\lambda^{2}-\left[\left(1-t_{1}\right) k_{1}+t_{1} k\right] \lambda+e^{-r \lambda} . \\
& f_{t_{2}}(\lambda)=\lambda^{2}-\left[\left(1-t_{2}\right) k_{1}+t_{2} k\right] \lambda+e^{-r \lambda}
\end{aligned}
$$

com $t_{1}, t_{2} \in[0,1]$. Temos que

$$
f_{t_{2}}(\lambda)=f_{t_{1}}(\lambda)+g(\lambda)
$$

$\operatorname{com} g(\lambda)=\lambda\left(k-k_{1}\right)\left(t_{1}-t_{2}\right)$.

Para aplicarmos o Teorema (1.2) resta mostrar que $|g(\lambda)|<\left|f_{t_{1}}(\lambda)\right|$ para $\lambda \in \partial U_{0 b\left(k_{2}\right)}$. 


\section{Temos}

$$
|g(\lambda)|=|\lambda|\left(k-k_{1}\right)\left|t_{1}-t_{2}\right|
$$

Tomando

$$
\left|t_{1}-t_{2}\right|<\frac{\min \left|f_{t_{1}}(\lambda)\right|}{\max |\lambda|\left(k-k_{1}\right)}
$$

para $t_{1} \in[0,1], \lambda \in \partial U_{0 b\left(k_{2}\right)}$ e $k \in\left[k_{1}, k_{2}\right]$, temos que $|g(\lambda)|<\left|f_{t_{1}}(\lambda)\right|$.

Logo, da compacidade de $[0,1]$ mostramos que o número de zeros de $f_{t}$ em $U_{0 b\left(k_{2}\right)}$ é constante para $t \in[0,1]$ e $k \in\left[k_{1}, k_{2}\right]$.

Como já mostramos que o número de zeros de $f_{0}(\lambda)=\lambda^{2}-k_{1} \lambda+e^{-r \lambda}$ é exatamente 2 em $U_{0 b\left(k_{2}\right)}$, temos que o número de zeros de $f_{1}(\lambda)=\lambda^{2}-k \lambda+e^{-r \lambda}$ é exatamente 2, para $k \in\left[k_{1}, k_{2}\right]$, com $k_{2}$ fixo. Logo, para cada $k>-k_{0}(r)$ existem precisamente duas raízes da equação (2.3) com $\Re(\lambda)>0$ e $-\pi / r<\Im(\lambda)<$ $\pi / r$.

Se $r>0$ for suficientemente pequeno afirmamos que existe $\bar{k}>0$ tal que, a equação (2.3) não tem raízes reais para $-1<k<\bar{k}$. Além disso, $k_{0} \in(-1, \bar{k})$. De fato, da equação (2.4), tomando $\sigma=0$, temos

$$
\mu^{2}-k \mu=-e^{-\mu r} \text {. }
$$

- Para $k<-k_{0}$, temos que todas as raízes tem parte real negativa e portanto sabemos o comportamento das soluções, da equação linear, isto é, a solução nula é globalmente assintoticamente estável.

Analizemos para $k>-k_{0}$.

- Se $-k_{0}<k<0$, para que a equação (2.6) tenha raiz real é necessário que

$$
\mu^{2}-k \mu=-1,
$$

tenha raiz real.

Pelo discriminante da equação acima vemos que para $k>-2$ não temos raízes reais. 
Mostremos que para $r$ suficientemente pequeno, $-k_{0}>-2$.

De acordo com o Lema (2.2),

$$
\sigma^{2}=\cos \sigma r
$$

portanto, da pequenez de $r$, temos que $|\sigma|$ é aproximadamente 1. Dessa maneira $1 / \sigma$ é limitado. Ainda pelo Lema (2.2),

$$
k_{0}=\left(\sigma_{0}\right)^{-1} \operatorname{sen} \sigma_{0} r
$$

onde $\sigma_{0}$ é a única raiz da equação (2.7) em $(0, \pi / 2 r)$. Assim, temos que $k_{0} \rightarrow 0$ quando $r \rightarrow 0$.

Logo,

$$
k_{0}<1
$$

ou seja,

$$
-k_{0}>-1>-2 \text {. }
$$

Assim para $-k_{0}<k<0$ a equação (2.6) não tem raiz real.

- Se $k=0$, é obvio da equação

$$
\mu^{2}=-e^{-\mu r}
$$

que não temos raízes reais de (2.6).

- Seja $k>0$. Observando que o membro esquerdo da equação (2.6) descreve uma parábola cujo valor mínimo é $-\left(k^{2}\right) / 4$ e que o membro direito independe de $k$, vê- se que existe $\bar{k}$, tal que para $0<k<\bar{k}$, não existem raízes reais da equação (2.6).

Para $k \geq \bar{k}$ aparecem raízes reais.

Concluimos que para $-1<k<\bar{k}$, se $r$ é suficientemente pequeno, não há raiz real da equação (2.6).

No que segue, $k$ será sempre considerado nesse intervalo. 
Teorema 2.1 A equação (2.1) tem uma Bifurcação de Hopf em $k=-k_{0}(r)$, onde $k_{0}(r)$ está definido no Lema (2.2).

Prova: Segue do Lema (2.2) e do Teorema(1.3). 


\section{Capítulo 3}

\section{Um Teorema de Periodicidade}

A seguir acrescentamos algumas hipóteses sobre $f$ e $g$ na equação (2.1) e provaremos que esta tem uma solução periódica não constante para todo $-k_{0}<k<\bar{k}$.

Embora esse resultado seja apenas existencial, ele completa o Teorema (2.1), pois a bifurcação de Hopf é local ao passo que o nosso resultado será global. Isto é, ao parâmetro $k$ será permitido variar arbitrariamente em $\left(-k_{0}, \bar{k}\right)$.

Suponhamos que:

(3 a) $F(x)=\int_{0}^{x} f(x) d x$ é impar.

(3 b) $F(x) \rightarrow \infty$ quando $|x| \rightarrow \infty$ e há um $\beta>0$ tal que $F(x)>0$ e é crescente para $x>\beta$.

(3 c) $g^{\prime}(x)>0, g(x)=-g(-x), g^{\prime}(0)=1$.

(3 d) $g\left(F^{-1}(x)\right) / x \rightarrow 0, F^{-1}(x) / x \rightarrow 0$ quando $x \rightarrow \infty$.

(3 e) $f^{\prime}(x)>0$ para $x>0$.

Observação: Uma condição suficiente para que (3 d) esteja satisfeita é que $g$ seja limitada e $F(x)>A x^{2}, A>0$, para $x>$ constante positiva. De fato, nestas condições $F^{-1}(x)<(1 / \sqrt{A}) \sqrt{x}, x>$ constante positiva. Portanto,

$$
F^{-1}(x) / x<\sqrt{1 / A x} \text { e } \lim _{x \rightarrow \infty} F^{-1}(x) / x=0 .
$$


Com as hipóteses acima, a equação (2.1) é equivalente ao sistema;

$$
\left\{\begin{array}{l}
\dot{x}(t)=y(t)-F(x(t)) \\
\dot{y}(t)=-g(x(t-r))
\end{array}\right.
$$

Seja $C_{0}=C([-r, 0], \mathbf{R}) \times \mathbf{R}$. Assim se $\psi \in C_{0}, \psi$ é da forma $\psi=(\phi, a)$, onde $\phi \in C([-r, 0], \mathbf{R})$ e $a \in \mathbf{R}$.

É util observar que o espaço $C_{0}$ pode ser naturalmente identificado ao subespaço $\tilde{C} \operatorname{de} C\left([-r, 0], \mathbf{R}^{2}\right)$ definido por

$$
\varphi=\left(\varphi_{1}, \varphi_{2}\right) \in \tilde{C} \Leftrightarrow \varphi_{2}(\theta) \equiv \text { constante },-r \leq \theta \leq 0 .
$$

Essa identificação é dada pela bijeção,

$$
\psi=(\phi, a) \in C_{0} \mapsto\left(\varphi_{1}, \varphi_{2}\right) \in \tilde{C},
$$

onde $\varphi_{1}=\phi, \varphi_{2}(\theta) \equiv a,-r \leq \theta \leq 0$.

Para cada $\psi \in C_{0}$, a equação (3.1) tem uma única solução $z(\psi), z=(x, y)$, com $z_{0}=\psi$. De fato, dado $\psi \in C_{0}, \psi=(\phi, a)$, a segunda equação do sistema (3.1) estará satisfeita por uma única função $y(t),-r \leq t \leq r$, definida por

$$
y(t)= \begin{cases}a & \text { se }-r \leq t \leq 0 \\ a-\int_{0}^{t} g(\phi(s-r)) d s & \text { se } 0 \leq t \leq r\end{cases}
$$

Substituindo $y(t)$ na primeira equação $\operatorname{com} t \geq 0$, obtemos uma equação diferencial ordinária em $x$

$$
\dot{x}=y(t)-F(x)
$$

que satisfaz todas as hipóteses do Teorema do Problema de Valor Inicial. Assim, definindo

$$
x(t)=\phi(t),-r \leq t \leq 0
$$

e $x(t)$ como a solução do problema de valor inicial

$$
\begin{aligned}
\dot{x} & =y(t)-F(x) \\
x(0) & =\phi(0)
\end{aligned}
$$


à direita de 0 , obtemos a única solução de $(3.1),(x(t), y(t))$, num intervalo $[-r, \tau), \tau>0$.

Os resultados que se seguem garantem o prolongamento dessa solução à $[-r, \infty)$

A aplicação $z_{t} \in C_{0}$ designará a solução da equação (3.1) onde $z_{t}=\left(x_{t}, y(t)\right)$.

Também usaremos as notações $z(t)=(x(t), y(t))$ e $\psi(0)=(\phi(0), a)$, se $\psi \in C_{\mathbf{0}}$.

Definamos o subconjunto de $C_{0}$ por

$$
K=\left\{\psi=(\phi, a) \in C_{0}: 0 \leq a<\infty, 0=\phi(-r) \leq \phi(\theta),-r \leq \theta \leq 0\right\}
$$

Lema 3.1 Se as hipóteses sobre $f$ e $g$ estão satisfeitas, então as seguintes afirmações valem:

(i) Há uma aplicação $\tau_{1}: K \backslash\{0\} \rightarrow(r, \infty)$ contínua tal que

$$
z_{\tau_{1}(\psi)}(\psi) \in-K \stackrel{\text { def }}{=}\{-\psi: \psi \in K\}
$$

(ii) Há uma aplicação contínua $\tau_{2}: K \backslash\{0\} \rightarrow(r, \infty)$ tal que $z_{\tau_{2}(\psi)}(\psi) \in K$.

(iii)Para cada $\psi \in K \backslash\{0\}$, a solução $z(\psi)$ da equação (3.1) é oscilatória, isto é, ambos $x(\psi)(t)$ e $y(\psi)(t)$ tem infinitos zeros.

Prova: Se $\psi \in K$ e $z(\psi)$ é solução da equação (3.1), então $-z(\psi)=z(-\psi)$.

Mostremos que $-z(\psi)=(-x(t),-y(t))$ é solução de (3.1).

Temos

$$
-y(t)-F(-x(t))=-y(t)+F(x(t))=-[y(t)-F(x(t))]=[-x(t)]^{0}
$$

e

$$
-g(-x(t-r))=g(x(t-r))=-[-g(x(t-r)]=[-y(t)] .
$$

Além disso,

$$
[-z]_{0}=-\psi
$$


Logo,pela unicidade de soluções $-z(\psi)=z(-\psi)$.

Portanto $(i) \Rightarrow(i i)$. Como (i) e (ii) implicam (iii) é necessário provar somente (i).

Essa prova será dividida em duas partes. A primeira mais simples, refere-se ao caso $k>0$ e basicamente, é uma adaptação da prova de Hale [4]. A segunda, referente a $k<0$, é ignorada em Hale [4].
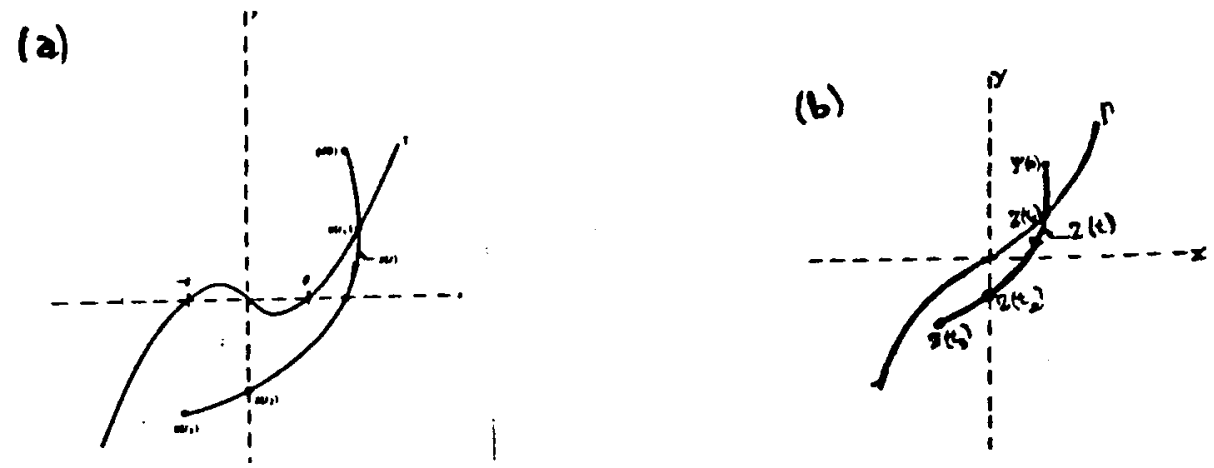

\{Figura 3.1 (a) $k>0$, (b) $k<0\}$

Suponhamos então $k>0$.

Se $\psi \in K$ e $z=z(\psi)$ é solução da equação (3.1) através de $\psi$, analizaremos a curva no plano $(x, y)$ traçada por $z(t), t \geq 0$. Seja

$$
\Gamma=\left\{(x, y) \in \mathbf{R}^{2} ; y=F(x), x \in \mathbf{R}\right\}
$$

Como $\psi \in K, \psi(0)$ está no primeiro quadrante. Suponhamos $\psi(0)$ acima de $\Gamma$.

Enquanto $z(\mathrm{t})$ está acima de $\Gamma$, temos $y(t)>F(x(t))$ e $x(t-r) \geq 0$ o que implica que $\dot{x}(t)>0$ e $\dot{y}(t) \leq 0$ por (3.1).

Mostremos que $z(t)$ intersepta $\Gamma$. Seja,

$$
Q=\{(x, y) ; x, y \geq 0 \text { e } y>F(x)\} .
$$

Lembremos que $\psi(0) \neq(0,0)$ e suponhamos temporariamente que $(x(t), y(t))$ pertença a $Q$, para $t>0$. Temos assim que $\mathrm{x}(\mathrm{t})$ é crescente e $\mathrm{y}(\mathrm{t})$ é decrescente. 
Logo, como ambas são limitadas, existem os limites

$$
\lim _{t \rightarrow \infty} x(t)=x_{0} \text { e } \lim _{t \rightarrow \infty} y(t)=y_{0} .
$$

Temos que $x_{0}>0$, pois $x(0)>0$ e $x$ é estritamente crescente. Além disso, existe sequência $\tau_{n} \rightarrow \infty$, tal que $\dot{y}\left(\tau_{n}\right) \rightarrow 0$, quando $n \rightarrow \infty$.

Como $\tau_{n} \rightarrow \infty$, segue-se que $\tau_{n}-r \rightarrow \infty$, quando $n \rightarrow \infty$. Assim, $x\left(\tau_{n}-r\right) \rightarrow$ $x_{0}$, quando $n \rightarrow \infty$. Pela continuidade da $g, g\left(x\left(\tau_{n}-r\right)\right) \rightarrow g\left(x_{0}\right)$. Da condição $\dot{y}\left(\tau_{n}\right) \rightarrow 0$, quando $n \rightarrow \infty$, combinada com

$$
\dot{y}\left(\tau_{n}\right)=-g\left(x\left(\tau_{n}-r\right)\right)
$$

tiramos que $g\left(x_{0}\right)=0$, ou seja, $x_{0}=0$, o que é uma contradição com o fato de ser $x_{0}>0$.

Portanto, existe um primeiro tempo $t_{1}$ tal que $z\left(t_{1}\right) \in \Gamma$. Assim $\dot{x}\left(t_{1}\right)=0 \mathrm{e}$ $\dot{y}\left(t_{1}\right)<0$, como decorre da equação (3.1).

Também, enquanto $z(\mathrm{t})$ está abaixo de $\Gamma$ e $x(t) \geq 0$, temos $y(t)<F(x(t))$ e $g(x(t-r)) \geq 0$ o que implica $\dot{x}(t)<0$ e $\dot{y}(t) \leq 0$.

0 próximo passo é mostrar que existe $t_{2}>0$ tal que $x\left(t_{2}\right)=0$. Para tanto, fazemos a afirmação: $z(\mathrm{t})$ não cruza $\Gamma$ para $t>t_{1}$ e $x(t) \geq 0$. Isto segue do fato que em qualquer desses cruzamentos $z(t)$ teria declividade vertical, o que é impossivel.

Portanto, há um $\delta>0$ e $\epsilon>0$ tal que $\dot{x}(t)<-\delta<0$ para $t>t_{1}+\epsilon$ e $x(t) \geq 0$. Assim, há um primeiro valor $t_{2}>t_{1}$ tal que $x\left(t_{2}\right)=0$ e $\dot{x}\left(t_{2}\right)<-\delta$.

Um argumento similar ao acima implica que existe $t=t_{4}, t_{4}>t_{2}$ tal que $z\left(t_{4}\right) \in \Gamma$ e $t_{2}<t \leq t_{4}$. Isso implica $\dot{y}\left(t_{4}\right)>0$ e $x\left(t_{4}-r\right)<0$.

Se $t_{3}=t_{2}+r$, então $z_{t_{3}} \in(-K)$, pois $x_{t_{3}}$ é decrescente e $x_{t_{3}}(-r)=0$. Se $\tau_{1}(\psi)=t_{3}$, então $\tau_{1}: K \backslash\{0\} \rightarrow(r, \infty)$ e $z_{\tau_{1}(\psi)} \in(-K)$. Mostremos que $\tau_{1}$ é contínua. 
Fixemos o intervalo $\left[0, t_{2}(\psi)+\alpha\right], \alpha>0$ fixo porém arbitrário. Pelo Teorema da Continuidade com relação as condições iniciais, temos que, para todo $\epsilon>0$, existe $\delta=\delta(\epsilon)$ tal que se $|\psi-\varphi|<\delta$ então $\left|z_{t}(\psi)-z_{t}(\varphi)\right|<\epsilon, t \in\left[0, t_{2}(\psi)+\alpha\right]$. Como estamos considerando a norma do sup temos que,

$$
|x(\psi)(t)-x(\varphi)(t)|<\epsilon, t \in\left[0, t_{2}(\psi)+\alpha\right]
$$

Escolhemos $\tilde{t}, \bar{t}$, e $\epsilon^{\prime}, 0<\epsilon^{\prime}<\epsilon$ de modo que $\tilde{t}$ e $\bar{t} \in\left(0, t_{2}(\varphi)+\alpha\right)$, da seguinte maneira:

$$
\begin{aligned}
& \tilde{t}=t_{2}(\varphi)-\epsilon^{\prime} \text { tal que } x(\varphi)(\tilde{t})>0 \\
& \bar{t}=t_{2}(\varphi)+\epsilon^{\prime} \text { tal que } x(\varphi)(\bar{t})<0
\end{aligned}
$$

Tomemos então $\psi_{n} \rightarrow \varphi$. Temos,

$$
\begin{aligned}
& x\left(\psi_{n}\right)(\tilde{t}) \rightarrow x(\varphi)(\tilde{t})>0 \\
& x\left(\psi_{n}\right)(\bar{t}) \rightarrow x(\varphi)(\bar{t})<0
\end{aligned}
$$

Como $x\left(t_{2}\left(\psi_{n}\right)\right)=0$, temos que existe $N$ tal que se $n \geq N$ então $t_{2}\left(\psi_{n}\right) \in$ $(\tilde{t}, \tilde{t})$.

Logo,

$$
\begin{aligned}
t_{2}(\varphi)-\epsilon^{\prime}<t_{2}\left(\psi_{n}\right)<t_{2}(\varphi)+\epsilon^{\prime} & \Rightarrow-\epsilon^{\prime}<t_{2}\left(\psi_{n}\right)-t_{2}(\varphi)<\epsilon^{\prime} \\
& \Rightarrow\left|t_{2}\left(\psi_{n}\right)-t_{2}(\varphi)\right|<\epsilon^{\prime}
\end{aligned}
$$

Portanto, $\psi_{n} \rightarrow \varphi \Rightarrow t_{2}\left(\psi_{n}\right) \rightarrow t_{2}(\varphi)$, ou seja, $t_{2}(\psi)$ é uma função contínua em $\psi$. Como $\tau_{1}(\psi)=t_{2}(\psi)+r$, temos $\tau_{1}(\psi)$ é contínua.

O caso $k<0$ é análogo ao anterior exceto por apresentar uma dificuldade maior em garantir - se que a solução cruza o eixo $x$ em um ponto de abscissa positiva. 
Apresentamos a seguir a prova desse fato. Para isto vamos analizar a solução abaixo da curva $\Gamma$ e acima do eixo $x$.

Observemos que devido $x$ e $y$ serem decrescentes nessa região, podemos tirar um único $y$ como função de $x$. Dessa maneira, temos $y=y(x(t))$, e pela regra da cadeia,

$$
\frac{d y}{d x}=\frac{d y}{d t} / \frac{d x}{d t}
$$

Suponhamos que a solução não cruzasse o eixo $x$, para $x>0$.

Temos que a solução $z(t)$ não passa por $(0,0)$ em um instante $\tilde{t}$, pois se passasse, nesse instante teríamos,

$$
\dot{x}(\tilde{t})=0
$$

e

$$
\dot{y}(\tilde{t})=-g(x(t-r))<0 .
$$

Dessa maneira o vetor tangente á curva em $(0,0)$, seria ortogonal ao eixo $x$. Pela hipótese ( 3 a) sobre $F$

$$
\frac{d F}{d x}(0)=-k<1
$$

ou seja, o ângulo que a reta tangente á curva $\Gamma$ em $(0,0)$ faz com o ejxo $x$ é menor que $\pi / 4$.

Logo, chegamos que a curva teria que estar acima de $\Gamma$, próximo de $(0,0), 0$ que é uma contradição.

Sendo assim, temos que $z(t) \rightarrow \tilde{z}=(\tilde{x}, \tilde{y})$ onde $\tilde{z}$ é um ponto de $\Gamma, \operatorname{com} \tilde{x}$, $\tilde{y}>0, z(t) \rightarrow(0,0)$ ou cruza o eixo $x$ quando $t \rightarrow \infty$.

Mostremos que o primeiro caso não ocorre. Caso contrário, $y(t) \rightarrow \tilde{y}$, ou seja, $\dot{y}\left(t_{n}\right) \rightarrow 0, n \rightarrow \infty$, ao longo de alguma sequência $t_{n}$, com $t_{n} \rightarrow \infty$, quando $n \rightarrow \infty$. 
Logo, pela equação ( 3.1$), g\left(x\left(t_{n}-r\right) \rightarrow 0\right.$.

Mas $x(t) \rightarrow \tilde{x}, t \rightarrow \infty$, logo $x(t-r) \rightarrow \tilde{x}$. Assim pela continuidade da $g$, temos que $g(x(t-r)) \rightarrow g(\tilde{x})$. Pela unicidade do limite $g(\tilde{x})=0$. Logo, $\tilde{x}=0$. Uma contradição.

Portanto o que temos é $z(t)=(x(t), y(t)) \rightarrow(0,0)$ quando $t \rightarrow \infty$.

Dado $\epsilon>0$ arbitrário, escolhemos $\xi_{0}$ de tal modo que

$$
F^{\prime}(\xi)<-k+\epsilon \text { e } g^{\prime}(\xi)>-k
$$

para $\xi \in\left[0, \xi_{0}\right]$.

Para $x \in\left[0, \xi_{0}\right]$, pelo Teorema do Valor Médio temos,

$$
g(x(t-r))=g^{\prime}(\xi) x(t-r)>-k x(t-r),
$$

e

$$
F(x(t))=F^{\prime}(\bar{\xi}) x(t)<(-k+\epsilon) x(t) .
$$

Dessa maneira, para $x_{0} \in\left[0, \xi_{0}\right]$,

$$
\begin{aligned}
\frac{d y}{d x}\left(x_{0}\right) & =\frac{g(x(t-r))}{F(x(t))-y(t)} \geq \frac{g(x(t-r))}{F(x(t))} \\
& >\frac{-k x(t-r)}{(-k+\epsilon) x(t)}>\frac{-k x(t)}{(-k+\epsilon) x(t)}=\frac{-k}{-k+\epsilon} .
\end{aligned}
$$

Quando $\epsilon \rightarrow 0, \frac{-k}{-k+\varepsilon} \rightarrow 1$ e $x_{0} \rightarrow 0$. Logo, $\frac{d y}{d x}(0) \geq 1$.

Isso leva a uma contradição com o fato de $\frac{d F}{d x}(0)<1$.

Desse modo $z(t) \nrightarrow(0,0)$, ou seja, a solução cruza o eixo $x$, para um ponto de abscissa positiva.

Se $\psi(0)$ está entre $\Gamma$ e o eixo $x$, o mesmo argumento pode ser usado para completar a prova do lema.

Para cada $\psi \in K \backslash\{0\}$, seja $\tau_{1}(\psi)$ o número dado pelo Lema (3.1), parte (i), e defina $A: K \rightarrow K$ por

$$
A \psi= \begin{cases}-z_{\tau_{1}(\psi)}(\psi) & \text { se } \psi \neq 0 \\ 0 & \text { se } \psi=0\end{cases}
$$


Se $\psi \neq 0$ é um ponto fixo de $A$, isto é, $A \psi=\psi$, então a simetria da equação (3.1) implica que

$$
z_{2 \tau_{1}(\psi)}(\psi)=z_{\tau_{1}(\psi)}\left(z_{\tau_{1}(\psi)}(\psi)\right)=z_{\tau_{1}(\psi)}(-\psi)=-z_{\tau_{1}(\psi)}(\psi)=\psi
$$

donde $\psi$ corresponde a uma solução periódica não trivial da equação ( 3.1 ) de período $2 \tau_{1}(\psi)$.

$O$ que resta mostrar portanto é a existência de um tal ponto fixo $\psi \in K \backslash\{0\}$.

Lema 3.2 A aplicação $A: K \backslash\{0\} \rightarrow K$ definida acima é completamente contínua.

Prova: Seja $B=\left\{\psi=(\phi, a) \in K \backslash\{0\}:|\phi(0)| \leq M_{0}, a \leq a_{0}\right\}$ um conjunto limitado arbitrário em $K \backslash\{0\}$.

Mostremos que o conjunto, $H=\left\{-z_{\tau_{1}(\psi)} ; \psi \in B\right\}$ é relativamente compacto.

Como $H=\left\{-\left(x_{\tau_{1}(\psi)}, y\left(\tau_{1}(\psi)\right) ; \psi \in B\right\}\right.$, se mostrarmos que as projeções de $H$ em cada coordenada são relativamente compactas, teremos que $H$ será relativamente compacto.

Para isso, mostraremos que o conjunto $H 1=\left\{-y\left(\tau_{1}(\psi)\right), \psi \in B\right\} \subset \mathbf{R}$ é limitado e usaremos o Teorema de Ascoli- Arzela para o conjunto $H 2=\left\{-x_{\tau_{1}(\psi)}\right.$; $\psi \in B\}$.

Mostremos, em primeiro lugar que $H 1$ e $H 2$ são limitados.

Consideremos $\xi\left(a_{0}\right)$ de modo que $a_{0}-F\left(\xi\left(a_{0}\right)\right)=0$ e $M>\max \left(M_{0}, \xi\left(a_{0}\right)\right)$.

Se $0 \leq t \leq t_{2}(\psi) \stackrel{\text { def }}{=} \tau_{1}(\psi)-r$, então $0 \leq x(t)<M$. Para $0 \leq t \leq t_{2}(\psi)+r$, temos, $-r \leq t-r \leq t_{2}(\psi)$. Assim, para $-r \leq t-r \leq 0,0 \leq x(t-r)=x_{t}(-r) \leq$ $x(0)<M$ e para $0<t-r \leq t_{2}(\psi)$ temos $0<x(t-r)<M$ pelo que foi feito anteriormente.

Logo,

$$
0 \leq-\dot{y}(t)=g(x(t-r))<g(M) ; 0 \leq t \leq t_{2}(\psi)+r
$$


Suponhamos que exista um $\tau \in\left(0, t_{2}(\psi)\right)$ tal que $F_{m}-y(\tau)=M / r$ onde $F_{m}=\inf \{F(x) ; 0 \leq x \leq M\}$. Temos,

$$
\dot{x}(t)=y(t)-F(x(t)) .
$$

Para $0 \leq x \leq M, F_{m} \leq F(x)$. Assim

$$
\dot{x}(t) \leq y(t)-F_{m}=-\left(F_{m}-y(t)\right) \leq-M / r,
$$

enquanto $\tau \leq t \leq \tau+r$ e $x(t) \geq 0$.

Afirmação: $\tau \leq t_{2}(\psi) \leq \tau+r$.

Suponhamos por absurdo que $\tau+r<t_{2}(\psi)$.

Como $\tau$ e $\tau+r \in\left(0, t_{2}(\psi)\right)$, temos que $0 \leq x(\tau)<M$ e $0 \leq x(\tau+r)<M$. Então pelo Teorema do Valor Médio

$$
x(\tau+r)-x(\tau)=\dot{x}(\xi) r, \tau \leq \xi \leq \tau+r .
$$

Temos que

$$
\dot{x}(\xi) \leq-M / r .
$$

Logo,

$$
x(\tau+r)-x(\tau) \leq-M
$$

ou seja,

$$
x(\tau)-x(\tau+r) \geq M,
$$

o que é um absurdo, pois tanto $x(\tau)$ como $x(\tau+r)$ são positivos e menores que $M$. Portanto, $x(\tau+r) \leq 0$, o que implica que $t_{2}(\psi) \leq \tau+r$. Assim, de

$$
-\dot{y}(t)=g(x(t-r)) \leq g(M), 0 \leq t \leq t_{2}(\psi)+r
$$

temos que,

$$
-y(t) \leq-y(\tau)+g(M)(t-\tau) .
$$


Logo,

$$
-y(t) \leq 2 r g(M)+M / r-F_{m} \stackrel{\text { def }}{=} y_{m}
$$

para $0 \leq t \leq t_{2}(\psi)+r$.

$\operatorname{Se} \beta=\inf \{s ; F(s)=0\}$, então $\beta \leq 0$. Se $x(t)>\beta$ para $t_{2}(\psi) \leq t \leq t_{2}(\psi)+r$, temos

$$
|x(t)| \leq \max \{M,|\beta|\}
$$

e fica provado que os conjuntos $H 1$ e $H 2$ são limitados.

Se há um $\tau \in\left[t_{2}(\psi), t_{2}(\psi)+r\right)$ tal que $x(\tau)=\beta$, então $\dot{x}(t) \geq y(t) \geq-y_{m}$ para $\tau \leq t \leq t_{2}(\psi)+r$. Portanto,

$$
x(t) \geq-y_{m} r+\beta
$$

e

$$
|x(t)| \leq \max \left\{M,|\beta|+\left|y_{m}\right| r .\right\} \stackrel{\text { def }}{=} x_{m} .
$$

Se não existe $\tau$, tal que $F_{m}-y(\tau)=M / r$, ou seja, $y(\tau)=-M / r+F_{m}$, então $y(t)<-M / r+F_{m}$ para $0 \leq t \leq t_{2}(\psi)+r$.

Portanto, fica demonstrado que $x(\mathrm{t})$ e $y(\mathrm{t})$ para $t \in\left[0, t_{2}(\psi)+r\right]$ são uniformemente limitados para $\psi \in B$.

Mostremos que $H 2$ é equicontínuo, ou seja, dado $\epsilon>0$ existe $\delta>0, \delta=\delta(\epsilon)$, tal que, $|\theta-\hat{\theta}|<\delta \Rightarrow\left|x_{\tau_{1}(\psi)}(\theta)-x_{\tau_{1}(\psi)}(\hat{\theta})\right|<\epsilon$, para $\psi \in B$.

Sem perda de generalidade podemos supor que $\hat{\theta} \leq \theta$.

Temos que,

$$
\begin{aligned}
x_{\tau_{1}(\psi)}(\theta)-x_{\tau_{1}(\psi)}(\hat{\theta}) & =x\left(\tau_{1}(\psi)+\theta\right)-x\left(\tau_{1}(\psi)+\hat{\theta}\right) \\
& =\dot{x}(\tau)(\hat{\theta}-\theta) \\
& =[y(\tau)-F(x(\tau))](\hat{\theta}-\theta),
\end{aligned}
$$

$\operatorname{com} \tau \in\left(\tau_{1}+\hat{\theta}, \tau_{1}+\theta\right)$. 
Foi mostrado anteriormente que

$$
|x(t)| \leq x_{m} \text { e }|y(t)| \leq y_{m}
$$

para $0 \leq t \leq t_{2}(\psi)+r$.

Da continuidade de $F$ segue-se que existe $L>0$ tal que $|F(x)|<L$ para $x \in\left[-x_{m}, x_{m}\right]$. Assim,

$$
|\dot{x}(\tau)| \leq|y(\tau)|+|F(x(\tau))|<y_{m}+L
$$

Portanto, dado $\epsilon>0$, tomando $\delta<\epsilon /\left(y_{m}+L\right)$, decorre da equação (3.3) que

$$
\left|x_{\tau_{1}(\psi)}(\theta)-x_{\tau_{1}(\psi)}(\hat{\theta})\right|<\epsilon
$$

para toda $\psi \in B$

Lema 3.3 Há uma constante $M>0$ tal que se $|\psi|=M, \psi \in K \backslash\{0\}$ e $A \psi=$ $\mu \psi$, então $\mu<1$.

Prova: Usaremos a notação do Lema (2.2).

Mostremos que se $\psi \in K \backslash\{0\}$ e $A \psi=\mu \psi \operatorname{com} \psi(0)$ abaixo de $\Gamma$, do fato de $(A \psi)(0)$ estar acima de $\Gamma$ segue - se que $\mu<1$.

Temos que $A \psi(0)=-z_{\tau_{1}(\psi)}(0)=-\left(x\left(\tau_{1}(\psi)\right), y\left(\tau_{1}(\psi)\right)\right)$. Como $-A \psi(0)=$ $\left(x\left(\tau_{1}(\psi)\right), y\left(\tau_{1}(\psi)\right)\right)$ está abaixo de $\Gamma$ e $F$ é impar, temos que $A \psi(0)$ está acima de $\Gamma$. Uma vez que $A \psi=\mu \psi$, temos $A \psi(0)=\mu \psi(0)$. Ou seja, $\psi(0)$ e $A \psi(0)$, estão sobre uma reta passando pela origem em $\mathbf{R}^{2}$.

Pela hipótese (3 e), para todo $M>\phi(0)$, se $|\psi|=M$, então $\mu<1$.

Suponhamos $\psi(0)$ acima de $\Gamma$. Para um dado $\alpha>0$ seja $\xi=\xi(\alpha)$ a solução positiva de $\alpha-F(\xi)=0$. Se $\psi=(\phi, a)$, a hipótese (3 e) implica que existe $x_{1}$ tal que $F(x)>x$, para $x>x_{1}$. Se tomarmos $M \geq \sqrt{x_{1}^{2}+F\left(x_{1}\right)^{2}}$, temos que para $|\psi|=M$ e $\psi(0)$ acima de $\Gamma,|\psi|=\max \{\phi(0), a\}=a$. 
Para $x(t) \geq 0$ e $\psi(0)$ acima de $\Gamma, x(t) \leq \xi(\mathrm{a})$ para $0 \leq t \leq t_{2}(\psi) \stackrel{\text { def }}{=} \tau_{1}(\psi)-r$. Assim, $0 \leq-\dot{y}(t) \leq g(\xi(a))$, para $0 \leq t \leq t_{2}(\psi)+r$. Suponhamos que existe $\tau \in\left(0, t_{2}(\psi)\right)$ tal que $F_{m}-y(\tau)=\xi(a)$ onde $F_{m}=\inf \{F(x) ; 0 \leq x \leq \xi(a)\}$. Então $\dot{x} \leq-\xi(a) / r$ enquanto $\tau \leq t \leq \tau+r$ e $x(t) \geq 0$. De maneira análoga ao Lema (3.2), concluimos que $\tau \leq t_{2}(\psi) \leq \tau+r$. Logo,

$$
-y(t) \leq 2 r g(\xi(a))+\xi(a) / r-F_{m} .
$$

Como $y\left(\tau_{1}(\psi)\right)<0$, temos $-y\left(\tau_{1}\right)=\left|y\left(\tau_{1}(\psi)\right)\right|$.

Assim,

$\left|y\left(\tau_{1}(\psi)\right)\right| \leq 2 r g(\xi(a))+\frac{\xi(a)}{r}+\left|F_{m}\right|=\left[\frac{2 r g(\xi(a))}{a}+\frac{F^{-1}(a)}{a r}+\frac{\left|F_{m}\right|}{a}\right] a$.

Pela hipótese (3 d), existe $a_{0}>0$ e $k<1$ tal que $\left|y\left(\tau_{1}(\psi)\right)\right| \leq k a<a$ para $a \geq a_{0}, \psi \in K$, com $\psi(0)$ acima de $\Gamma$.

Lembrando que $y\left(\tau_{1}(\psi)\right)$ é a ordenada de $A \psi(0)=\mu \psi(0)$, isso garante que $\mu<1$. Em outros termos,

$$
A \psi=\mu \psi \Rightarrow A \psi(0)=\mu \psi(0)=\mu(\phi(0), a)
$$

e

$$
A \psi=-z_{\tau_{1}(\psi)} \Rightarrow A \psi(0)=-z_{\tau_{1}(\psi)}(0)=-\left(x\left(\tau_{1}(\psi)\right), y\left(\tau_{1}(\psi)\right) .\right.
$$

Logo,

$$
\mu a=-y\left(\tau_{1}(\psi)\right)<a .
$$

Portanto,

$$
\mu<1 \text {. }
$$

Para darmos continuação à análise da equação (2.1), vamos precisar da decomposição do espaço de fase em soma direta, $C_{0}=P_{\lambda} \oplus Q_{\lambda}$, associada ao 
autovalor $\lambda$ de (2.3). Uma completa descrição desses fatos é encontrado em Hale [[4], cap7]. Ressaltamos aqui, somente alguns fatos que serão importantes para $o$ nosso problema específico.

De acordo com o Lema (2.2), podemos selecionar um autovalor simples $\lambda$ de (2.3) , com $\Re(\lambda)>0$. Lembrando que os auto valores da equação (2.3) ocorrem aos pares conjugados, podemos assumir também, $0<\Im(\lambda)<\pi / r$.

Temos que a parte linear das equações (3.1) é;

$$
\left\{\begin{array}{l}
\dot{x}(t)=y(t)+k x(t) \\
\dot{y}(t)=-x(t-r)
\end{array}\right.
$$

Seja

$$
L(\phi)=\left(\begin{array}{ll}
k & 1 \\
0 & 0
\end{array}\right) \phi(0)+\left(\begin{array}{rr}
0 & 0 \\
-1 & 0
\end{array}\right) \phi(-r)
$$

o campo vetorial do sistema (3.4).

Sua representação de Riesz é dada por uma matriz $2 \times 2, \eta(\theta)$, definida para $\theta \in[-r, 0]$, como

$$
\begin{aligned}
\eta(-r) & =-\left(\begin{array}{cc}
k & 1 \\
-1 & 0
\end{array}\right) \\
\eta(\theta) & =-\left(\begin{array}{ll}
k & 1 \\
0 & 0
\end{array}\right) ;-r \leq \theta<0 \\
\eta(0) & =0 .
\end{aligned}
$$

Ou seja, por simples cálculos de rotina pode -se ver que

$$
L \phi=\int_{-r}^{0}[d \eta(\theta)] \phi(\theta)
$$

para $\phi \in C_{0}$.

Seja $\mathrm{G}$ ser $\circ$ gerador infinitesimal do semigrupo $\mathrm{T}(\mathrm{t}), t \geq 0$, definido pelas soluções de (3.4), isto é, $\mathrm{T}(\mathrm{t}) \phi=z_{t}(., \phi), \phi \in C_{0}, t \geq 0$. Então, $\lambda$ é um autovalor simples de $\mathrm{G}$ e o subespaço $P_{\lambda}$ é precisamente o auto-espaço generalizado unidimensional associado a $\lambda, M_{\lambda}(G)$. 
Se $G^{*}$ é o operador adjunto formal de G, $\lambda$ é também um autovalor de $G^{*}$ e seu autoespaço generalizado $M_{\lambda}\left(G^{*}\right)$ é unidimensional. Seja $C_{0}^{*}=C([0, r], \mathbf{R}) \times \mathbf{R}$, onde seus elementos são entendidos como vetores linha.

A forma bilinear de $C_{0}^{*} \times C_{0}$, dada por

$$
<\psi, \phi>=\psi(0) \phi(0)-\int_{-r}^{0} \int_{0}^{\theta} \psi(\xi-\theta)[d \eta(\theta)] \phi(\xi) d \xi
$$

onde $\psi \in C_{0}^{*}, \phi \in C_{0}$ aparece de maneira natural na decomposição de $C_{0}$ em soma direta de subespaços invariantes realizada em Hale [[4], cap 7]. Ali também aparece, na definição da equação caracteristica a função

$$
\Delta(\lambda)=\lambda I-\int_{-r}^{0} e^{\lambda \theta}[d \eta(\theta)]
$$

Se $u$ é uma solução não trivial de $\Delta(\lambda) u=0$, e $v$ um vetor linha, uma solução não trivial de $v \Delta(\lambda)=0$, então as funções $\rho(t)=e^{\lambda t} u, t \in[-r, 0]$ e $\xi(s)=v e^{-\lambda s}$, $s \in[0, r]$, geram $M_{\lambda}(G)$ e $M_{\lambda}\left(G^{*}\right)$, respectivamente. A decomposição de $C_{0}=$ $P_{\lambda} \oplus Q_{\lambda}$, onde $P_{\lambda}$ e $Q_{\lambda}$ são invariantes sob o operador solução $\mathrm{T}(\mathrm{t})$ definido acima, escrevemos cada $\phi \in C_{0}$ como $\phi=\phi^{P_{\lambda}}+\phi^{Q_{\lambda}}$. A componente $\phi^{P_{\lambda}}$ de $\phi \in C_{0}$ em $P_{\lambda}$, ou seja, $\pi_{\lambda}(\phi)=\phi^{P_{\lambda}}$, onde $\pi_{\lambda}$ é a projeção associada a $P_{\lambda}$, é dada por,

$$
\phi^{P_{\lambda}}=<\xi, \phi>\rho
$$

$\mathrm{Na}$ formulação do próximo lema, o número $\tau_{1}(\psi), \psi \in K \backslash\{0\}$, é o mesmo definido no lema (3.1).

Lema 3.4 Se $k_{0}(r)$ é como no Lema (2.2), então para $k>-k_{0}$ e $\delta>0$,

$$
\inf \left\{\left|\pi_{\lambda}\left(z_{t}\right)\right|: z_{t}=z_{t}(., \psi), \psi \in K,|\psi|=\delta, 0 \leq t \leq \tau_{1}(\psi)\right\}>0
$$

Prova: De acordo com o que colocamos acima, sendo $z_{t}=z_{t}(., \psi), \pi_{\lambda}\left(z_{t}\right)=$ $z_{t}^{P_{\lambda}}=\left\langle\xi, z_{t}>\rho\right.$, para todo $\psi \in C_{0}$, onde $\rho$ e $\xi$, são bases de $M_{\lambda}(G)$ e $M_{\lambda}\left(G^{*}\right)$, 
respectivamente. Assim,

$$
\left|\pi_{\lambda}\left(z_{t}\right)\right|=\left|<\xi, z_{t}>\| \rho\right|
$$

Como $\rho$ é base, tem-se $|\rho| \neq 0$, logo, podemos nos restrigir à forma bilinear $<\xi, z_{t}>$, para todo $\psi \in K \cap \partial B_{6}$.

Vamos encontrar uma base $\xi$ para $M_{\lambda}\left(G^{*}\right)$. Temos que,

$$
\begin{aligned}
\Delta(\lambda) & =\lambda\left(\begin{array}{ll}
1 & 0 \\
0 & 1
\end{array}\right)-\int_{-r}^{0} e^{\lambda \theta}\left(\begin{array}{ll}
d \eta_{11} & d \eta_{12} \\
d \eta_{21} & d \eta_{22}
\end{array}\right) \\
& =\lambda\left(\begin{array}{ll}
1 & 0 \\
0 & 1
\end{array}\right)-\left(\begin{array}{rr}
k & 1 \\
-e^{-\lambda r} & 0
\end{array}\right) \\
& =\left(\begin{array}{rr}
\lambda-k & -1 \\
e^{-\lambda r} & \lambda
\end{array}\right) .
\end{aligned}
$$

Logo se $\xi(s)=v e^{-\lambda s}$ e $v=\left(v_{1}, v_{2}\right)$ então $v$ deve satisfazer,

$$
\left(v_{1}, v_{2}\right)\left(\begin{array}{cr}
\lambda-k & -1 \\
e^{-\lambda r} & \lambda
\end{array}\right)=0
$$

ou seja,

$$
\left\{\begin{aligned}
(\lambda-k) v_{1}+e^{-\lambda r} v_{2} & =0 \\
-v_{1}+\quad \lambda v_{2} & =0
\end{aligned}\right.
$$

Se $v_{2}=1$, então, $v_{1}=\lambda$.

Dessa maneira, uma base para $M_{\lambda}\left(G^{*}\right)$ é

$$
\xi(s)=e^{-\lambda s}(\lambda, 1)
$$

Determinemos agora a forma bilinear de $C_{0}^{*} \times C_{0}$, calculada no par $\left(\xi, z_{t}\right)$, onde $z_{t}=\left(x_{t}, y(t)\right)$ :

$$
\begin{aligned}
<\xi \quad & z_{t}>= \\
& =\xi(0) z_{t}(0)-\int_{-\tau}^{0} \int_{0}^{\theta} \xi(\alpha-\theta)[d \eta(\theta)] z_{t}(\alpha) d \alpha \\
& =(\lambda, 1)\left(\begin{array}{l}
x(t) \\
y(t)
\end{array}\right)-\int_{-\tau}^{0} \int_{0}^{\theta}(\lambda, 1) e^{-\lambda(\alpha-\theta)}\left[\begin{array}{ll}
d \eta_{11} & d \eta_{12} \\
d \eta_{21} & d \eta_{22}
\end{array}\right]\left(\begin{array}{l}
x(t+\alpha) \\
y(t)
\end{array}\right) d \alpha
\end{aligned}
$$




$$
\begin{aligned}
= & \lambda x(t)+y(t)-\int_{-r}^{0} \int_{0}^{\theta}\left(\lambda d \eta_{11}+d \eta_{21}\right) e^{-\lambda(\alpha-\theta)} x(t+\alpha) d \alpha \\
& -\int_{-r}^{0} \int_{0}^{\theta}\left(\lambda d \eta_{12}+d \eta_{22}\right) y(t) e^{-\lambda(\alpha-\theta)} d \alpha \\
= & \lambda x(t)+y(t)-\int_{-r}^{0} e^{-\lambda(\alpha+r)} x(t+\alpha) d \alpha .
\end{aligned}
$$

Tomando $\lambda=\gamma+i \sigma$, com $\gamma>0$ e $0<\sigma<\pi / r$, temos

$$
<\xi, z_{t}>=\gamma x(t)+y(t)+i \sigma x(t)-\int_{-r}^{0} e^{-\lambda(\alpha+r)} x(t+\alpha) d \alpha
$$

Daqui em diante dividiremos a prova em dois casos: $k>0$ e $k \leq 0$.

1o caso: $k>0$.

Para esse caso, vamos considerar a mudança de variável, $t=r \tau$, ou seja, definamos $X(\tau), Y(\tau)$ por:

$$
\left\{\begin{array}{l}
X(\tau)=x(r \tau) \\
Y(\tau)=y(r \tau)
\end{array}\right.
$$

e $Z(\tau)=(X(\tau), Y(\tau))$, com $Z_{\tau}=\left(X_{\tau}, Y(\tau)\right)$.

O nosso sitema já linearizado passa a ser

$$
\left\{\begin{array}{l}
\dot{X}(\tau)=r(Y(\tau)+k X(\tau)) \\
\dot{Y}(\tau)=-r X(\tau-1)
\end{array}\right.
$$

Considerando o vetor linha $(\lambda / r, 1)$, obtemos uma base $\xi(s)=e^{-\lambda s}(\lambda / r, 1)$ e a forma bilinear em $C_{0}^{*} \times C_{0}$

$$
<\xi, Z_{\tau}>=\frac{\lambda}{r} X(\tau)+Y(\tau)-r \int_{-1}^{0} e^{-\lambda(\alpha+1)} X(\tau+\alpha) d \alpha
$$

para $0 \leq \tau \leq \tau_{1} / r$, ou ainda,

$$
<\xi, z_{t}>=\gamma \frac{x(t)}{r}+y(t)+i \sigma \frac{x(t)}{r}-r \int_{-1}^{0} e^{-\lambda(\alpha+1)} x(t+\alpha) d \alpha
$$

para $0 \leq t \leq \tau_{1}$

Analizaremos a forma bilinear acima para $r$ suficientemente pequeno e $0 \leq t \leq \tau_{1}$ 
Tomando $B_{\delta}=\{\psi \in K \backslash\{0\}:|\psi|=\delta\}$ e $M>\max \left(F^{-1}(\delta), \delta\right)$, a prova de que $x(t), t \in\left[0, \tau_{1}(\psi)\right]$ é limitada, é análoga à prova de que $H_{1}$ e $H_{2}$ são limitados no Lema (3.2). Dessa maneira, temos que

$$
r \int_{-1}^{0} e^{-\lambda(\alpha+1)} x(t+\alpha) d \alpha \rightarrow 0
$$

quando $r \rightarrow 0$.

Tomando $\lambda=\gamma+i \sigma$, com $\gamma>0$ e $0<\sigma<\pi / r$, resta analizar o número complexo

$$
\gamma \frac{x(t)}{r}+y(t)+i \sigma \frac{x(t)}{r} .
$$

Para isto será importante a construção de um retângulo no quarto quadrante com lados apoiados sobre os eixos, independente da condição inicial, $\psi \in$ $K \backslash\{0\} \cap \partial B_{\delta}$, onde as soluções $Z(\psi)$ não entram.

Mostremos que exitem $m, q$ tal que se $\psi \in K \backslash\{0\} \cap B_{\delta}$ então $z(t) \notin$ $\{0 \leq x \leq q, m \leq y \leq 0\}$.

Chamemos de n o ponto de interseç̧ão da solução com o eixo $\mathrm{x}$. Temos dois casos a considerar: $n \geq \beta$ e $n<\beta$, onde $\beta>0$ é a abscissa do ponto de intersecção de $\Gamma$ com o eixo $x$.

(a) $n \geq \beta$.

Tomemos nesse caso $\mathrm{m}$ o ponto de mínimo de $\mathrm{F}$ para $0 \leq x \leq \beta$ e $q=$ $F^{-1}(m)$.

(b) $n<\beta$.

Lembrando que nossas condições iniciais $\psi$ satisfazem $\psi(0)=(\phi(0), a)$, podemos ter $a \leq \delta$. Assim,

- Se $a<\delta$, tomemos $q=\delta$ e $m=F(q)$.

- Se $a=\delta$, do sistema obtido de (3.1) através da mudança de variável 


$$
\left\{\begin{array}{l}
\dot{x}(t)=r(y(t)-F(x(t)) \\
\dot{y}(t)=-r g(x(t-1))
\end{array}\right.
$$

temos que

$$
|\dot{y}(t)| \leq r L, \quad 0 \leq t \leq 1 .
$$

Pelo Teorema do Valor Médio,

$$
|y(1)-y(0)|=|\dot{y}(\xi)|, \quad 0 \leq \xi \leq 1 .
$$

Assim, como $\dot{y}(t) \leq 0$, para $t \in[0,1]$, temos:

$$
r L \geq-[y(1)-y(0)]=a-y(1),
$$

donde

$$
y(1) \geq \delta-r L .
$$

Para $r$ suficientemente pequeno, temos, $y(1) \geq \delta-r L>\delta / 2>0$. Como $\dot{y}(t) \leq 0$ para $0 \leq t \leq 1, y(t) \geq y(1) \geq \delta / 2$.

Logo,

$$
\dot{x}(t)=r(y(t)-F(x(t)) \geq r y(t)>r \delta / 2 .
$$

Pelo Teorema do Valor Médio, existe algum $\eta \in(0,1)$ tal que

$$
x(1)-x(0)=\dot{x}(\eta)>r \delta / 2 .
$$

Logo,

$$
x(1) / r>\delta / 2>0 .
$$

Podemos então tomar $q=\delta / 2$ e $\mathrm{m}=\mathrm{F}(\mathrm{q})$.

Passamos então a analizar a expressão

$$
\gamma \frac{x(t)}{r}+y(t)+i \sigma \frac{x(t)}{r} .
$$

Isto será feito em 3 casos. 
(a) $x(t) \geq 0$ e $y(t)>0$, que por sua vez, é subdividido nos casos: $a<\delta$ e $a=\delta$.

- Se $a<\delta$, então necessariamente $\phi(0)=\delta$, donde

$$
\frac{x(t)}{r} \geq \min \{\delta, \beta\} .
$$

Logo, basta olhar para a parte imaginária da expressão (3.6).

- Se $a=\delta$ então $x(0) \leq \delta$.

Para $0 \leq t \leq 1$, tem-se $x(t-1) \leq x(0)$. Assim,

$$
g(x(t-1)) \leq g(\delta)=\eta>0 .
$$

Uma vez que

$$
\dot{y}(t)=-r g(x(t-1))
$$

temos

$$
0>\dot{y}(t) \geq-r \eta
$$

Tomemos $r$ suficientemente pequeno tal que $r \eta<\delta / 2$.

Pelo Teorema do Valor Médio

$$
y(1)-y(0)=\dot{y}(\xi)
$$

para $\xi \in[0,1]$, ou seja,

$$
y(1) \geq-r \eta+\delta>-\delta / 2+\delta=\delta / 2 .
$$

Lembrando que $y$ é uma função decrescente nessa região, vem

$$
y(t) \geq y(1) \geq \delta / 2,
$$

para $0 \leq t \leq 1$. Logo, para $0 \leq t \leq 1$, basta olhar para a parte real de (3.6), ou seja,

$$
\gamma \frac{x(t)}{r}=y(t) \geq \frac{\delta}{2} .
$$


Para $t>1$, temos,

- Se $z(t)$ cruza $\Gamma$ no primeiro quadrante

$$
\frac{x(t)}{r} \geq \min \left\{\frac{\delta}{2}, \beta\right\}
$$

- Se $z(t)$ não cruza $\Gamma$ no primeiro quadrante

$$
\frac{x(t)}{r} \geq \frac{\delta}{2}
$$

pois $x(1) / r \geq \delta / 2$ e $x(t)$ cresce enquanto $y(t)>F(x(t))$.

Assim para $t>1$, basta olhar para a parte imaginária de (3.6).

Dessa maneira,

$$
k \xi, z_{t}>1>\overline{k_{1}}>0 \text {. }
$$

enquanto $z(t)$ permanecer no primeiro quadrante.

(b) $x(t) \geq 0$ e $y(t) \leq 0$.

Seja o retângulo $\{0 \leq x \leq q e m \leq y \leq 0\}$ construido anteriormente $\mathrm{e}$ chamemos de $s$ a reta $y=-\gamma x / r$. Tomemos $r$ suficientemente pequeno, de maneira que a reta $s$ intersepta o retângulo para $y=m$. Dessa maneira, esse ponto é $\left(\frac{-r m}{\gamma}, m\right)$.

- Se $0 \leq x(t) / r \leq-m / 2 \gamma$, então,

$$
\gamma \frac{x(t)}{r}+y(t) \leq \frac{-m}{2}+m=\frac{m}{2}<0 .
$$

- Se $x(t) / r>-m / 2 \gamma$, então

$$
\sigma \frac{x(t)}{r}>\frac{-m \sigma}{2 \gamma}>0 .
$$

Portanto,

$$
\left|<\xi, z_{t}>\right|>\overline{k_{2}}>0 .
$$


(c) $x(t)<0$ e $y(t)<0$.

Temos $y(t)<m<0$. Logo,

$$
\gamma \frac{x(t)}{r}+y(t)<m<0 .
$$

Portanto,

$$
k \xi, z_{t}>1>\overline{k_{3}}>0 \text {. }
$$

Como $z(t)$ ser solução da equação (3.1) é equivalente $z(t r)$ ser solução da equação (3.5), de (a),(b),(c) concluimos que

$$
\inf \left\{\left|<\xi, z_{t}(\psi)>\right|, \psi \in K,|\psi|=\delta, 0 \leq t \leq \tau_{1}(\psi)\right\}>\min \left\{\overline{k_{1}}, \overline{k_{2}}, \overline{k_{3}}\right\}>0
$$

2o Caso : $k \leq 0$.

Como já foi salientado anteriormente, para os nossos propósitos é suficiente considerarmos $k>-1$.

Seja $Q=\{(x, y): 0 \leq x, y \leq \delta, \max (x, y)=\delta\}$ e $\psi \in K$ tal que $\psi(0)=$ $(x(0), y(0)) \in Q$.

Seja $p_{1}$ a abscissa do ponto de interseç̧ão da solução com a curva $\Gamma$.

Subdividiremos a análise deste caso, nos casos em que $z(t)$ está acima de $\Gamma$ e depois abaixo.

Seja,

$$
G(\tilde{\sigma}, \delta)=\inf \left\{p_{1}(\psi): \psi \in K, \psi(0)=(\tilde{\sigma}, \delta), \forall(\tilde{\sigma}, \delta) \in Q \text { e } \delta \geq F(\delta)\right\}
$$

Temos que $\tilde{\sigma} \geq 0$.

(i) $\tilde{\sigma}>0$.

Analizando a equação (3.1), temos que nessa região onde está definido $\mathrm{G}$, $x$ é uma função crescente. Assim, $p_{1}(\psi)>\tilde{\sigma}>0$, para todo $\psi \in K$, com $\psi(0)=(\tilde{\sigma}, \delta) \in Q$. 
Logo,

$$
G(\tilde{\sigma}, \delta) \geq \tilde{\sigma}>0 .
$$

(ii) $\tilde{\sigma}=0$, ou seja, $\psi(0)=(0, \delta) \in Q$.

Seja,

$$
\xi= \begin{cases}\delta & \operatorname{se} F(\delta) \geq \delta \\ F^{-1}(\delta) & \operatorname{se} F(\delta)<\delta .\end{cases}
$$

Se $x(r)<\xi / 2, \dot{x}(t)=y(t)-F(x(t))>\delta / 2$ para $0 \leq t \leq r$. Então, pelo Teorema do Valor Médio, temos,

$$
x(r)=\dot{x}(\bar{t}) r>(\delta / 2) r .
$$

Portanto,

$$
x(t)>\min \{\xi / 2, \delta / 2 r\}
$$

para $r \leq t \leq t_{1}(\psi)$, onde $t_{1}(\psi)$ é o instante de cruzamento da solução com a curva $\Gamma$.

Dessa maneira, $p_{1}(\psi)>\min \{\xi / 2, \delta / 2 r\}$, para todo $\psi \in K,(x(0), y(0)) \in Q$. Assim, também nesse caso, $G(\tilde{\sigma}, \delta)>0$.

Resta analizar, abaixo da curva.

Será importante na análise da forma bilinear, mostrarmos que para qualquer $\psi \in K,|\psi|=\delta$ a abscissa do ponto de cruzamento da solução correspondente com o eixo $\mathrm{x}$, é maior que algum número estritamente positivo. Assim, esse é o nosso próximo passo.

Tomemos $\epsilon>0$ e $\xi=\xi(\epsilon)$, tal que $-k+\epsilon<1, F^{\prime}(x)<-k+\epsilon$ e $g^{\prime}(x)>-k$ para $x \in[0, \xi]$.

Se a solução, $z(t)=(x(t), y(t))$ cruza a reta $x=\xi$, de acordo com a relação (3.2), na prova do lema (3.1), temos que y pode ser escrito como função de $x$ e, para $x_{0} \in(0, \xi]$,

$$
\frac{d y}{d x}\left(x_{0}\right)>\frac{-k}{-k+\epsilon}>-k .
$$


Tomemos $\bar{\xi}$ tal que se $0 \leq x \leq \bar{\xi}$, então $F^{\prime}(x)<-k /(-k+\epsilon)$.

Seja, $\xi_{0}=\min \{\xi, \bar{\xi}\}$.

Chamemos de $\ell$ a reta que passa por $\left(\xi_{0}, F\left(\xi_{0}\right)\right)$ e possui declividade $-k /(-k+\epsilon)$. E seja $z_{1}$ e $z_{2}$ os pontos de interseção da curva solução $z(t)$ com a reta $x=\xi_{0}$ e $\ell$, respectivamente.

Pelo Teorema do Valor Médio existe um ponto $\tilde{z}$ de $z(t)$, onde a reta tangente por $\tilde{z}$ é paralela ao segmento de reta que une $z_{1}$ e $z_{2}$.

Essa reta possui declividade menor que $-k /(-k+\epsilon)$. O que nos leva á uma contradição com o fato que $d y / d x\left(x_{0}\right)>-k /(-k+\epsilon)$, para $x_{0} \in\left(0, \xi_{0}\right]$.

Concluimos assim, que toda solução cruza o eixo $x$, á direita de $\tilde{\xi}_{0}$, onde $\tilde{\xi}_{0}$ é o ponto de interseç̧ão de $\ell$ com o eixo $x$.

Analisemos a forma bilinear para $r$ suficientemente pequeno, e $t \in\left[0, \tau_{1}(\psi)\right]$.

$$
<\xi, z_{t}>=\gamma x(t)+y(t)+i \sigma x(t)-\int_{-r}^{0} e^{-\lambda(\alpha+r)} x(t+\alpha) d \alpha
$$

Analogamente ao caso $k>0$, vemos que $x$ é limitada. Assim,

$$
\int_{-\tau}^{0} e^{-\lambda(\alpha+r)} x(t+\alpha) d \alpha \rightarrow 0
$$

quando $r \rightarrow 0$.

Resta então analisar a expressão

$$
\gamma x(t)+y(t)+i \sigma x(t)
$$

(a) $x>0$ e $y \geq 0$.

- Se $y(t) \geq F(x(t))$ então $x(t) \geq \tilde{\sigma}$. Logo,

$$
\frac{x(t)}{r} \geq \frac{\tilde{\sigma}}{r}>\tilde{\sigma}
$$

para $r<1$. 
- Se $y(t)<F(x(t))$ então $x(t)>\tilde{\xi}_{0}$. Logo,

$$
\frac{x(t)}{r}>\frac{\tilde{\xi}_{0}}{r}>\tilde{\xi}_{0}
$$

para $r<1$.

(b) $x \geq 0$ e $y<0$.

Análogo ao caso $k>0$, construiremos um retângulo no quarto quadrante onde as soluções não entram.

Seja $\tilde{t_{1}}$ tal que $z\left(\tilde{t_{1}}\right)=\left(\tilde{\xi}_{0}, \eta\right)$ onde $\eta<0$.

Dado $\epsilon>0$, suponhamos $y(t)>-\epsilon$ onde $t \in\left[\tilde{t_{1}}, \tilde{t_{1}}+2 r\right]$ para $r$ suficientemente pequeno.

Temos

$$
|\dot{x}(t)| \leq F\left(\tilde{\xi}_{0}\right)+\epsilon=M
$$

se $r$ é suficientemente pequeno e $t \in\left[\tilde{t_{1}}, \tilde{t_{1}}+2 r\right]$.

Dessa maneira,

$$
\left|x\left(\tilde{t_{1}}+2 r\right)-x\left(\tilde{t_{1}}\right)\right|=|\dot{x}(\tau)| 2 r \leq 2 r M
$$

para $\tau \in\left[\tilde{t_{1}}, \tilde{t_{1}}+2 r\right]$.

Devido ao fato de $\dot{x}(t)$ ser negativo para $t \in\left[\tilde{t_{1}}, \tilde{t_{1}}+2 r\right]$, vem

$$
x\left(\tilde{t_{1}}+2 r\right) \geq x\left(\tilde{t_{1}}\right)-2 r M=\tilde{\xi_{0}}-2 r M>\tilde{\xi_{0}} / 2
$$

Logo,

$$
x(t)>\tilde{\xi}_{0} / 2
$$

para $t \in\left[\tilde{t_{1}}, \tilde{t_{1}}+2 r\right]$ e $r$ suficientemente pequeno.

Pelo Teorema do Valor Médio, para $\tau \in\left[\tilde{t_{1}}, \tilde{t_{1}}+2 r\right]$, temos

$$
y\left(\tilde{t_{1}}+2 r\right)-y\left(\tilde{t_{1}}+r\right)=\dot{y}(\tau) r=-g(x(\tau-r)) r \leq g\left(\tilde{\xi_{0}} / 2\right) r
$$


ou seja,

$$
y\left(\tilde{t_{1}}+2 r\right) \leq-g\left(\tilde{\xi_{0}} / 2\right) r=\tilde{M}
$$

Concluimos que

$$
x\left(\tilde{t_{1}}+2 r\right)>\xi_{0} / 2 \text { e } y\left(\tilde{t_{1}}+2 r\right)<\tilde{M}
$$

Seja $\bar{M}=\max (-\epsilon, \tilde{M})$. Temos que as soluções não entram no retângulo, $\left\{0 \leq x \leq \tilde{\xi}_{0} / 2\right.$ e $\left.\bar{M} \leq y \leq 0\right\}$

Consideremos a reta $y=-\gamma x(t)$ e o retângulo $R$, construido anteriormente. Seja o retângulo $\{0 \leq x<h$ e $w<y \leq 0\}$, obtido pela interseção da reta acima com o retângulo $R$.

Análogo ao caso $k>0$ concluimos que

$$
\left|<\xi, z_{t}>\right|>k_{2}>0
$$

(c) $y<0$ e $x<0$.

Como $y<w$,

$$
\gamma x(t)+y(t)<w<0
$$

Assim,

$$
\left|<\xi, z_{t}>\right|>k_{3}>0
$$

Concluimos com isso que

$\inf \left\{\left|<\xi, z_{t}(\psi)>1, \psi \in K,\right| \psi \mid=\delta, 0 \leq t \leq \tau_{1}(\psi)\right\} \geq \min \left\{k_{1}, k_{2}, k_{3}\right\}>0$.

Para concluirmos que 0 é ponto ejetivo de $A$, falta verificarmos a quarta hipótese do Teorema (1.6). Essa condição implica que $A$ é contínua em 0 e portanto, juntamente com o Lema (3.2), que $A: K \rightarrow K$ é completamente contínua.

Damos abaixo uma idéia da prova dessa hipótese sugerida em [4, pag 267]. 
Como nossos autovalores são complexos devido ao intervalo que consideramos $k,-1<k<\bar{k}$, concluimos que a aplicação $\tau_{1}$ é completamente contínua. Com isso, se $|\psi|<\delta$ então $\tau_{1}(\psi) \leq T$ para $T>0$ e $\psi \in K \backslash\{0\}$. Seja $\tau>T$. Pelo Teorema de Existência, Unicidade e Dependência contínua com relação às condições inicjais, dado $\epsilon>0$ existe $\tilde{\delta}$ tal que se $|\psi|<\tilde{\delta}$ então $\left|x_{t}(., \psi)\right|<\epsilon$ para $0 \leq t \leq \tau$.

Teorema 3.1 Se F e g satisfazem as condições (3 a)-(3f) e $f(0)<k_{0}(r)$ onde $k_{0}(r)>0$ é dado no Lema (2.2), então a equação (2.1) tem uma solução periódica $n \tilde{a} o$ constante.

Prova: Tomando $X=C_{0}$ e observando que o nosso conjunto $K \subset C_{0}$ é de dimensão infinita, os resultados anteriores mostram que o nosso operador $A$ satisfaz todas as hipóteses do Teorema (1.5). Portanto $A$ tem um ponto fixo $\psi \neq 0$. Ou seja, a equação (2.1) tem uma solução periódica não constante.

Daremos a seguir um exemplo onde $F$ e $g$ satisfazem as condições do Teorema (3.1).

$\mathrm{Na}$ equação

$$
\ddot{x}(t)+k\left(x^{2}(t)-1\right) \dot{x}(t)+x(t-r)=0,
$$

onde $k>0$, seja $g(x)=x$. Temos que $f(x)=k\left(x^{2}-1\right)$.

Verifiquemos as hipóteses sobre $F$ e $g$.

(a) $F(x)=k\left(x^{3} / 3-x\right)$. Dessa maneira $F$ é ímpar.

(b) $F(x) \rightarrow \infty$ quando $|x| \rightarrow \infty$ e $\beta=\sqrt{3}$ satisfaz as condições que $F(x)>0$ e monótona crescente para $x>\beta$.

(c) As hipóteses sobre $g$ estão obviamente satisfeitas.

(d) Aplicando L 'Hospital para $Q=F^{-1}(x) / x$ vemos que $Q \rightarrow 0$ quando $x \rightarrow \infty$. Dessa maneira,

$$
\frac{g\left(F^{-1}\right)}{x}=\frac{F^{-1}(x)}{x} \rightarrow 0
$$


quando $x \rightarrow \infty$.

(e) $f^{\prime}(x)=2 k x>0$ para $x>0$.

Concluimos que a equação (3.7), a qual é a equação de van der Pol com retardamento $r$, tem uma solução periódica não constante. 


\section{Bibliografia}

[1] Chow, S. N. and Hale, J. K.- Periodic solutions of autonomous equations. J. Math. Anal. Appl., 66(1978), 495-506.

[2] Conway, J.B.-Function of One Complex Variable. New York, 1978.

[3] Grafton, R.- A periodicity theorem for autonomouus functional differential equations. J. Differential Equations, 6(1969), 87-109.

[4] Hale, J. K.- Theory of Functional Differential Equation. New York, 1977

[5] Nussbaum, R-Periodic solutions of some nonlinear autonomous functional differential equations. Ann. Math. Pura Appl., 10(1974), 263-306.

[6] Táboas, P. Z.- Periodic solutions of a planar delay equation. Proceedings of the Royal Society of Ediburgh, 116A(1990), 85-101. 